\title{
Sick sinus syndrome aggravated by carbamazepine therapy for epilepsy
}

\author{
K.A. Hewetson, A.E.S. Ritch and R.D.S. Watson \\ Dudley Road Hospital, Birmingham B18 7QH, UK.
}

\begin{abstract}
Summary: A case of sick sinus syndrome aggravated by carbamazepine prescribed for epilepsy is described. A 70 year old woman with previously stable psychomotor epilepsy experienced syncope of increased frequency after her anticonvulsant was changed to carbamazepine. ECG monitoring confirmed a severe sick sinus syndrome which improved on carbamazepine withdrawal. Failure to distinguish carbamazepine-induced cardiac syncope from epileptic attacks may lead to an increase in dosage and aggravation of syncope.
\end{abstract}

\section{Introduction}

Carbamazepine-induced cardiac conduction abnormalities have only been described in patients being treated for trigeminal neuralgia. We report a case of cardiac syncope due to the sick sinus syndrome aggravated by carbamazepine prescribed for psychomotor epilepsy.

\section{Case report}

A 70 year old woman was admitted following a syncopal episode at home. Twenty years earlier she presented with generalized tonic-clonic seizures, vacant spells and a transient right homonymous hemianopia. The only abnormality detected was a left sided temporal focus on the electroencephalogram. Treatment with pheneturide for psychomotor epilepsy relieved her symptoms. Three years before admission carbamazepine $200 \mathrm{mg}$ t.d.s. was substituted and she began to experience vacant spells associated with pallor. She had lost consciousness on two occasions. She had also been taking diazepam $2 \mathrm{mg}$ b.d. and imipramine $50 \mathrm{mg}$ nocte for 14 years for depression. On admission she had congestive cardiac failure and a nodal bradycardia rate of 44 /minute, QRS duration 0.08 seconds. The serum carbamazepine concentration was raised at $15 \mathrm{mg} / 1$ (therapeutic range $2-10 \mathrm{mg} / \mathrm{l})$. Thyroid function tests were normal. Continuous 24 hour electrocardiogram (ECG) monitoring showed sinus node dysfunction with sinus pauses lasting up to 4.5 seconds (Figure 1). Diuretics were introduced and the other drugs discontinued. Three

Correspondence: K.A. Hewetson, M.B., M.R.C.P. Accepted: 1 November 1985 days later the maximum duration of sinus pauses was reduced to 2 seconds when a simultaneous serum carbamazepine level was $1.4 \mathrm{mg} / 1$. Further ECG monitoring when no drug was detectable in the blood showed a single short lived episode of sinus bradycardia of 36 beats/minute associated with a sinus pause of 1.8 seconds. No further vacant spells or episodes of cardiac syncope occurred and pacing was not required. She had a single tonic-clonic seizure 3 days after drug withdrawal.

\section{Discussion}

Steiner (1970) showed that carbamazepine prolonged atrio-ventricular conduction time and suppressed ventricular ectopic activity in dogs, but appeared to have no effect on intra-atrial or intra-ventricular conduction. Complete heart block (Beerman et al., 1975; Boesen et al., 1983), sinus bradycardia (Herzberg, 1978), and nodal bradycardia (Byrne et al., 1979) have been reported in patients receiving carbamazepine for trigeminal neuralgia at doses of $400 \mathrm{mg}, 1,000 \mathrm{mg}$ and $800 \mathrm{mg}$ daily, respectively. Initially it was thought that carbamazepine only aggravated pre-existing cardiac conduction abnormalities. However, Boesen et al. (1983) reported intermittent sino-atrial block and syncope in two patients who were taking doses of $600 \mathrm{mg}$ and $400 \mathrm{mg}$ carbamazepine daily and whose pre-treatment ECGs were normal.

Our patient had evidence of a mild sick sinus syndrome which was aggravated by carbamazepine prescribed for epilepsy. The duration of sinus pauses decreased as the serum concentration of carbamazepine fell from levels initially above the 


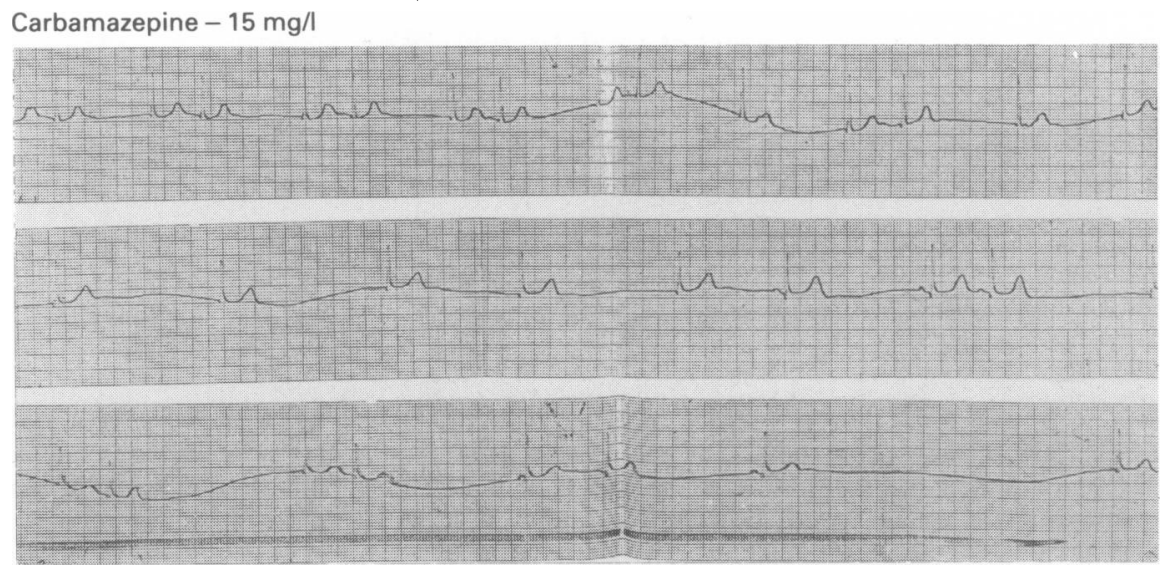

Figure 1 Twenty four hour electrocardiogram recording during treatment with carbamazepine. (Carbamazepine concentration $15 \mathrm{mg} / \mathrm{l}$.)

therapeutic range. Imipramine has been reported to affect atrio-ventricular conduction but it is unlikely to have been solely responsible as treatment was started 14 years earlier. However, interaction with carbamazepine can not be completely excluded.

No previous reports of cardiac conduction abnormalities have been reported in patients receiving carbamazepine for epilepsy. Boesen et al. (1983) suggest that the lack of reports involving epileptics may reflect the fact that the dose of carbamazepine is adjusted more gradually than in trigeminal neuralgia.

\section{References}

BEERMAN, B., EDHAG, O. \& VALLIN, H. (1975). Advanced heart block aggravated by carbamazepine. British Heart Journal, 37, 668.

BOESEN, F., ANDERSEN, E.B., JENSEN, E.K. \& LADEFORGED, S.D. (1983). Cardiac conduction disturbances during carbamazepine therapy. Acta Neurologica Scandinavica, 68, 49.

BYRNE, E., WONG, C.H., CHAMBERS, D.G. \& RICE, J.P.
However, a further reason may be the difficulty in distinguishing between epileptic seizures and cardiac syncope. We would suggest that every patient receiving carbamazepine for epilepsy who experiences an increase in the frequency of seizures or a change in their character should have continuous 24 hour ECG monitoring and an estimate of serum carbamazepin\& concentraton. Indeed, failure to recognize the de? velopment of cardiac syncope may lead to an increase in carbamazepine dosage with the consequent? aggravation of symptoms.

(1979). Carbamazepine therapy complicated by nodal bradycardia and water intoxication. Australia and New Zealand Journal of Medicine, 9, 295.

HERZBERG, L. (1978). Carbamazepine and bradycardia. Lancet, i, 1097.

STEINER, C. (1970). The anti-arrhythmic action of carbamazepine (Tegretol). Journal of Pharmacology and Experimental Therapeutics, 173, 323. 